\title{
NORMAL STRUCTURES FOR LOCALLY FLAT EMBEDDINGS
}

\author{
KENNETH C. MILLETT
}

1. Introduction. C. P. Rourke and B. J. Sanderson [4] have constructed a locally flat embedding of $S^{19} \times I$ in $S^{29}$ which does not have a normal microbundle [3]. Thus we are led to inquire into the extent to which the local flatness gives rise to some normal structure. In analogy with the development of normal structures for the differentiable case, we define several possible normal structures which are related to the normal microbundle and show that the RourkeSanderson example admits none of them. From this we conclude that the homotopy normal bundle [2] is the strongest normal structure available, in general. We also give an example of interest in the theory of Euclidean bundles.

Before beginning we warn the reader that these, rather natural, definitions are not in agreement with those currently used for topological (and piecewise linear) manifolds, e.g. a tubular neighborhood is usually called a normal bundle.

2. Definitions and statement of results. A Euclidean bundle (or an $\left(R^{n+k}, R^{n}\right)$-bundle) is a Steenrod fiber bundle whose fiber is $R^{n+k}$ and whose topological group is $H_{0}(n+k ; n)$, the group of homeomorphisms of $R^{n+k}$ which leave 0 and the hyperplane, $R^{n} \times 0$, invariant. Let $\tau_{M}$ denote the tangent $\left(R^{n}, 0\right)$-bundle of the topological manifold $M^{n}$ (of dimension $n$ ). If $f: M^{n} \rightarrow N^{n+k}$ is a locally flat embedding, $f^{*} \tau_{N}$ admits the structure of an $\left(R^{n+k}, R^{n}\right)$-bundle, $\left(f^{*} \tau_{N}, \tau_{M}\right)$, by $[2,4.3]$. A normal bundle for $f, \nu$, is a splitting of $\left(f^{*} \tau_{N}, \tau_{M}\right)$, i.e. an equivalence of $\left(f^{*} \tau_{N}, \tau_{M}\right)$ with $\left(\tau_{M} \oplus \nu, \tau_{M}\right)$. A tubular neighborhood for $f$ is an $\left(R^{k}, 0\right)$-bundle, $\nu=\left(E_{\nu}, p_{\nu}, M\right)$, and an embedding, $F$, of $E_{\nu}$ in $N$ such that $F \sigma_{0}=f$, where $\sigma_{0}$ denotes the zero section of $\nu$.

We note that, by $[2,4.11]$, a tubular neighborhood, $(\nu, F)$, gives a normal bundle for $f$, i.e. an equivalence between $\left(f^{*} \tau_{N}, \tau_{M}\right)$ and $\left(\tau_{M} \oplus \nu, \tau_{M}\right)$.

The homotopy normal bundle of $f$ always exists, and is defined as in [2]. That is, if $(\alpha, \beta)$ is an $\left(R^{n+k}, R^{n}\right)$-bundle over $X$, we define $E_{\nu(\alpha, \beta)}$ to be $\left(E_{\alpha} \backslash E_{\beta}\right) \cup \sigma_{0}(X)$ and $p_{\nu(\alpha, \beta)}$ to be the restriction of $p_{\alpha}$. The homotopy normal bundle of a locally flat embedding, $f$, is $\nu\left(f^{*} \tau_{N}, \tau_{M}\right)$. We may, then, ask if there is a Euclidean bundle which is fiber homotopy equivalent, in some appropriate sense, to the homotopy

Presented to the Society August 29, 1968; received by the editors January 29, 1968. 
normal bundle. Such a bundle would be called a homotopy normal Euclidean bundle. Thus, if $\alpha$ is a bundle with "zero" section, let $\alpha^{\prime}$ denote the bundle obtained by removing the image of the section. Two bundles with "zero" sections, $\alpha$ and $\beta$, over $X$ are said to be fiber homotopy equivalent if there exist fiber maps

$$
f:\left(E_{\alpha}, E_{\alpha^{\prime}}, \sigma_{0}(X)\right) \leftrightarrow\left(E_{\beta}, E_{\beta^{\prime}}, \sigma_{0}(X)\right): g
$$

such that $f g$ and $g f$ are homotopic to the appropriate identity maps, through homotopies of the triples which respect the fiber structure.

From its definition, it is clear that a normal bundle is a homotopy normal Euclidean bundle and hence we have defined a sequence of successively stronger normal structures, beginning with the homotopy normal bundle, which always exists, and ending with the tubular neighborhood, which need not exist. The main result of this paper concerns the others.

THEOREM 1. The Rourke-Sanderson locally flat embedding of $S^{19} \times I$ in $S^{29}$ does not have a homotopy normal Euclidean bundle.

A fortiori, Theorem 1 gives a locally flat embedding which has neither a normal bundle nor, as proved by Rourke and Sanderson, [4], a tubular neighborhood.

Question: Are there locally flat embeddings which distinguish the various normal structures?

Since the embedding does not have a normal bundle, there is a Euclidean bundle which does not split, i.e.

Example 2. There is an $\left(R^{29}, R^{20}\right)$-bundle over $S^{19}$ which does not split.

3. Proof of Theorem 1. This section is devoted to proving an extension of Theorem A, given by Rourke and Sanderson [4]. Since the properties of the example follow from this result in precisely the same way that their properties follow from Theorem A we do not include the remainder of the argument. With the exception of the notion of a quasi-link, and its properties, the notation and definitions used here are given in Rourke and Sanderson.'

Let $\xi$ be an $R^{q+1}$-bundle over $X$, i.e. $\xi$ is a fiber bundle, in the sense of Steenrod, whose fiber is $R^{q+1}$ and whose group is $H(q+1)$, the topological group of homeomorphisms of $R^{q+1}$. If $\xi$ is a numerable $R^{q+1}$-bundle, e.g. $X$ is paracompact, then it has a section [1, Corollary 2.8]. Furthermore, using the techniques developed in [1], one can prove the following lemmas. 
Lemma 1. Suppose $\xi$ is a numerable $R^{a+1}$-bundle over $X$ and $\sigma_{1}$ and $\sigma_{2}$ are two sections. Then there is a fiber isotopy

$$
\begin{aligned}
F: E_{\xi} & \times I \longrightarrow E_{\xi} \\
& \downarrow\left(p_{\xi}, 1_{I}\right) \quad \downarrow p_{\xi} \\
p r_{1}: X & \times I \longrightarrow X
\end{aligned}
$$

such that $F(x, 0)=x$ and $F\left(\sigma_{1}(x), 1\right)=\sigma_{2}(x)$.

Lemma 2. Suppose $\xi$ is a numerable $R^{q+1}$-bundle, and

$$
p_{\xi} \times 1_{I}: E_{\xi} \times I \rightarrow X \times I
$$

has sections, $\sigma_{1}$ and $\sigma_{2}$, such that $p r_{1} \circ \sigma_{1}(x, 0)=p r_{1} \circ \sigma_{1}(x, 1)$ $=p r_{1} \circ \sigma_{2}(x, t), t \in I$. Then there is a fiber isotopy taking $\sigma_{1}$ to $\sigma_{2}$ which is the identity on $E_{\xi} \times\{0,1\}$.

An $R^{a+1}$-bundle is said to be fiber homotopically trivial if its associated $\left(R^{q+1}, 0\right)$-bundle, which is well defined by Lemma 1 , is fiber homotopically trivial, via a trivialization, $t$. We define a quasi-link to be a quadruple, $\left(\xi, \sigma_{1}, \sigma_{2} ; t\right)$, where $\xi$ is a fiber homotopically trivial $R^{q+1}$-bundle over $S^{n}$, via $t$, and $\sigma_{1}$ and $\sigma_{2}$ are two noncoincident sections.

Paralleling [4], we next define quasi-linking classes and show that they are invariant under isotopy of quasi-links. Thus, let $\left(\xi, \sigma_{1}, \sigma_{2} ; t\right)$ be a quasi-link and let $\xi_{i}, i=1,2$, denote the associated $\left(R^{q+1}, 0\right)$ bundle having "zero section," $\sigma_{i}$. Finally, let $\sigma_{0}$ be the section of $\xi$ implicit in the definition of the fiber homotopy trivialization, $t$. By Lemma 1 there are fiber isotopies, $F_{i}$, such that $F_{i}\left(\sigma_{i}(x), 1\right)=\sigma_{0}(x)$. The first quasi-linking class of $\left(\xi, \sigma_{1}, \sigma_{2} ; t\right)$ is defined to be the element $\alpha_{1} \in \pi_{n}\left(s^{q}\right)$ determined by

$$
S^{n} \stackrel{\sigma_{1}}{\longrightarrow} E_{\xi_{2^{\prime}}} \stackrel{F_{2}(, 1)}{\longrightarrow} E_{\xi_{0^{\prime}}} \stackrel{t}{\longrightarrow} E_{\varepsilon^{q+1}} \stackrel{p r_{2}}{\longrightarrow} R^{q+1} \backslash 0 \stackrel{\rho}{\longrightarrow} S^{q} .
$$

The second quasi-linking class is defined similarly and the pair $\left(\alpha_{1}, \alpha_{2}\right)$ is called the quasi-linking class of $\left(\xi, \sigma_{1}, \sigma_{2} ; t\right)$. That it is well defined follows from Lemma 2, since any two definitions of $F_{2}$, for example, give rise to homotopic sections in $E_{\xi_{0}}$.

An isotopy of quasi-links is defined to be a continuous family of quasi-links or, equivalently, noncoincident sections to $E_{\xi} \times I$ over $S^{n} \times I$, say $\sigma_{1}^{\prime}$ and $\sigma_{2}^{\prime}$, which have the desired restriction to $S^{n} \times\{0,1\}$. By Lemma $1, \sigma_{2}^{\prime}$ is isotopic to $\sigma_{0} \times 1_{I}$, via $F$, so that the first quasilinking classes are equal, i.e. the maps into $S^{q}$ are homotopic via

$$
\rho \circ p r_{2} \circ t^{\prime} \circ F(, 1) \circ \sigma_{1}^{\prime}: S^{n} \times I \rightarrow S^{q},
$$


where $t^{\prime}$ is a fiber homotopy trivialization of $E_{\xi} \times I$ whose associated section is $\sigma_{0} \times 1_{I}$. Similarly, one shows that the second quasi-linking classes are equal and hence quasi-linking classes are invariant under isotopy.

Conversely, we note that two quasi-links are isotopic if and only if either the first or second quasi-linking classes are the same. Thus isotopy classes of quasi-links reflect some of the nature of the bundle. This is the motivation behind the proof of the following lemma, which is essentially the same as that given for Theorem A in [4].

LEMMA 3. If the first quasi-linking class of $\left(\xi^{9+1}, \sigma_{1}, \sigma_{2} ; t\right)$ is zero, $q$ odd $\geqq 3$, then the second quasi-linking class is of order two.

Proof. Without loss of generality, we may assume that $t$ has been chosen so that $\sigma_{0}=\sigma_{2}$ and, with respect to a trivialization, $g$, of $\xi^{q+1}$ over $S_{+}^{n}, \sigma_{1}(x)=g^{-1}(x, 1)$ and $t$ is $g$.

Using Lemma 1 and [1] we can construct a third section, $\sigma_{-1}$, which intersects neither $\sigma_{1}$ nor $\sigma_{0}$ and which is given by $g^{-1}(x,-1)$ with respect to the trivialization over $S_{+}^{n}$. Furthermore, it has the property that the pair of sections, $\left(\sigma_{1}, \sigma_{0}\right)$, is isotopic to $\left(\sigma_{0}, \sigma_{-1}\right)$, by an isotopy which is given by translation with respect to the trivialization over $S_{+}^{n}$. Indeed, $\sigma_{-1}$ is constructed via this translation and an extension to $\xi^{q+1}$ using [1].

The quasi-links, $\left(\xi, \sigma_{1}, \sigma_{0} ; t\right)$ and $\left(\xi, \sigma_{0}, \sigma_{-1} ; t\right)$ are isotopic and hence both have quasi-linking class $(0, \beta)$. To show that $\beta$ has order two it is necessary to define two auxiliary quasi-links.

Let $h: S^{n} \rightarrow S^{q}$ be a map such that $h\left(S_{-}^{n}\right)=(1,0, \cdots, 0)$ and $[h]=\beta$. Define $\lambda_{\mathbf{1}}$ and $\lambda_{-\mathbf{1}}$ by

$$
\begin{aligned}
\lambda_{1}(x) & =\sigma_{1}(x) & & \text { if } x \in S_{-}^{n}, \\
& =g^{-1}(x, h(x)) & & \text { if } x \in S_{+}^{n} ; \\
\lambda_{-1}(x) & =\sigma_{-1}(x) & & \text { if } x \in S_{-}^{n}, \\
& =g^{-1}(x,-h(x)) & & \text { if } x \in S_{+}^{n} .
\end{aligned}
$$

Using the isotopy taking $\left(\sigma_{1}, \sigma_{0}\right)$ to $\left(\sigma_{0}, \sigma_{-1}\right)$ we can show that the quasi-links, $\left(\xi, \lambda_{1}, \sigma_{0} ; t\right)$ and $\left(\xi, \sigma_{0}, \lambda_{-1} ; t\right)$, are isotopic and hence both have quasi-linking class $(\beta, 2 \beta)$.

Since the second quasi-linking classes of $\left(\xi, \sigma_{0}, \sigma_{-1} ; t\right)$ and $\left(\xi, \sigma_{0}, \lambda_{1} ; t\right)$ are both $\beta$, the quasi-links are isotopic. Thus $(0, \beta)=(2 \beta, \beta)$ and $2 \beta=0$.

TheOREM $\mathrm{A}^{\prime}$. Let $\mathrm{g}: \Sigma^{n} \times I^{1} \rightarrow R^{n+q+1}, q$ odd $\geqq 3$, be a piecewise linear embedding such that 
(a) g has a homotopy normal Euclidean bundle, $\eta$,

(b) $g \mid: \Sigma^{n} \times\{0\} \rightarrow R^{n+q+1}$ is the standard inclusion,

(c) $g \mid: \Sigma^{n} \times\{1\} \rightarrow R^{n+r+1} \backslash \Sigma^{n}$ is null homotopic.

Then the second linking class of $\left(g\left(\Sigma^{n} \times\{1\}\right), \Sigma^{n}\right)$ is of order two.

Proof. First extend $g$ to a piecewise linear embedding of $\Sigma^{n}$ $\times(-\infty,+\infty)$ into $R^{n+q+1} . \eta$ is also extended to $\Sigma^{n} \times(-\infty,+\infty)$. Let $\iota$ denote the standard inclusion of $\Sigma^{n}$ in $R^{n+q+1}$ and let $\sigma_{i}$ denote the embedding of $\Sigma^{n}$ in $\Sigma^{n} \times(-\infty,+\infty)$, which is identified with $E\left(\epsilon^{1}\right)$, given by $\sigma_{i}(x)=(x, i)$.

Since $\eta$ is fiber homotopy equivalent to $\nu_{g}$, the homotopy normal bundle of $g$, there is a fiber homotopy equivalence of $\xi=\epsilon^{1} \oplus \sigma_{0}^{*} \eta$ with $\epsilon^{1} \oplus \sigma_{0}^{*} \nu_{g}$ which is the identity on $\epsilon^{1}$. Let $\epsilon^{q+1}$ denote the standard tubular neighborhood of $\iota\left(\Sigma^{n}\right)$. It has a fiber homotopy trivialization defined by the map of $E_{\epsilon q+1^{\prime}}$ to $S^{q}$ arising from the inclusion of $E_{\epsilon q+1^{\prime}}$ in $\Sigma^{n+q+1} \backslash \Sigma^{n}$ and the retraction of $\Sigma^{n+q+1} \backslash \Sigma^{n}$ to $\Sigma^{q}$. By Theorem 4.7 of [2], $\epsilon^{1} \oplus \sigma^{*} \nu_{g}$ is fiber homotopy equivalent to $\epsilon^{q+1}$. Thus $\xi$ is fiber homotopy equivalent to $\epsilon^{q+1}$, via the composition of the fiber homotopy equivalences given above.

With this fiber homotopy trivialization of $\xi$, the quasi-linking class of $\left(\xi, \sigma_{i}, \sigma_{j} ; t\right), i \neq j$, equals the linking class of $\left(g \circ \sigma_{i}, g \circ \sigma_{j}\right)$. To prove this it is sufficient to consider only first classes, with $j=0$, as every quasi-linking class can be so represented. We chose the fiber homotopy equivalence of $\epsilon^{1} \oplus \sigma_{0}^{*} \nu_{g}$ with $\epsilon^{q+1}$ by passing through $\nu_{l}$, the homotopy normal bundle of the standard inclusion of $\Sigma^{n}$ in $\Sigma^{n+q+1}$. Because, under the inclusion of the fibers of $\nu_{\text {c }}$ into $\Sigma^{n+q+1} \backslash \Sigma^{n}, \sigma_{i}(x)$ goes to $g \circ \sigma_{i}(x)$, the maps defining the first classes are homotopic as maps into $\Sigma^{n+q+1} \backslash \Sigma^{n}$ and hence define the same first classes.

Since the quasi-linking class of $\left(\xi, \sigma_{1}, \sigma_{0} ; t\right)$ agrees with the linking class of $\left(g \circ \sigma_{1}, g \circ \sigma_{0}\right)$, it is sufficient to show that the second quasilinking class has order two. This follows from Lemma 3.

\section{REFERENCES}

1. A. Dold, Partitions of unity in the theory of fibrations, Ann. of Math. (2) 78 (1963), 223-255.

2. K. C. Millett, Homotopy normal bundles for locally flat immersions and embeddings of topological manifolds, Illinois J. Math. (to appear).

3. J. Milnor, Microbundles. I, Topology 3 (1964), Suppl. 1, 53-80.

4. C. P. Rourke and B. J. Sanderson, An embedding without a normal microbundle, Invent. Math. 3 (1967), 293-299.

Massachusetts Institute of Technology 